\title{
ARTIGO ORIGINAL \\ Cultura de segurança do paciente em unidades de terapia intensiva: percepção de profissionais de saúde
}

\author{
Patient safety culture in intensive care units: the perception of health professionals
}

\author{
Ana Lívia Araújo Girão (D, Anna Jessyca Andrade Lacerda² (D), Luana Sousa de Carvalho ${ }^{3}$ (D)

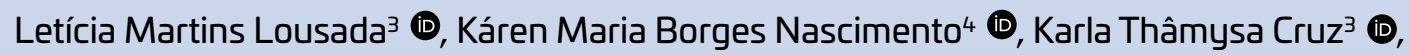 \\ Rhanna Emanuela Fontenele Lima de Carvalho ${ }^{3}$ (D)
}

\section{RESUMO}

Objetivou-se avaliar a percepção da cultura de segurança do paciente sob a perspectiva de profissionais de Unidades de Terapia Intensiva. Trata-se de um estudo transversal realizado com 283 profissionais de saúde de hospitais de referência do estado do Ceará. Os dados foram coletados por meio do Safety Attitudes Questionnaire, o qual busca avaliar as atitudes de segurança no exercício profissional. No escore total do questionário, entre os hospitais, observou-se variação de valores entre 63,4 a 71,5, sendo considerado valor positivo escore igual ou maior que 75. Portanto, nenhuma instituição alcançou resultados positivos. Entre os seis domínios do questionário, "Clima de segurança”, "Percepção da gerência” e "Condiçóes de trabalho" apresentaram as médias mais baixas. Desta forma, constatou-se a necessidade de incentivo à cultura de segurança em diversos aspectos, principalmente nas atitudes gerenciais quanto à segurança do paciente e condiçóes de trabalho.

Descritores: Segurança do Paciente; Unidades de Terapia Intensiva; Enfermagem de Cuidados Críticos; Cultura; Equipe de Assistência ao Paciente.

\section{ABSTRACT}

The objective of this study was to evaluate how patient safety culture is perceived by professionals working in Intensive Care Units. This is a cross-sectional study carried out with 283 health professionals from reference hospitals in the state of Ceará. The data collection used the Safety Attitudes Questionnaire, which seeks to evaluate safety attitudes in professional activity. Among hospitals, the questionnaire showed scores ranging from 63.4 to 71.5 , a positive score being equal to or greater than 75 . Therefore, no institution achieved positive results. Among the six domains of the questionnaire, "Safety climate", "Management perception" and "Working conditions" presented the lowest averages. Thus, this research verified the need to encourage safety culture in several aspects, mainly regarding managerial attitudes towards patient safety and working conditions.

Descriptors: Patient Safety; Intensive Care Units; Critical Care Nursing; Culture; Patient Care Team.

\footnotetext{
${ }^{1}$ Instituto Doutor José Frota - Fortaleza (CE), Brasil. E-mail: livia.girao@uece.br

${ }^{2}$ Graduação em Enfermagem, Universidade Estadual do Ceará - Fortaleza (CE), Brasil. E-mail: jessyca 3011@hotmail.com

${ }^{3}$ Universidade Estadual do Ceará - Fortaleza (CE), Brasil. E-mails: luanasousa741@gmail.com, leticia.lousada@hotmail.com, karlathamysa@hotmail.com, rhanna.lima@uece.br

${ }^{4}$ Hospital Geral de Fortaleza - Fortaleza (CE), Brasil. E-mail: kambn@hotmail.com
}

Como citar este artigo: Girão ALA, Lacerda AJA, Carvalho LS, Lousada LM, Nascimento KMB, Cruz KT, et al. Cultura de segurança do paciente em unidades de terapia intensiva: percepção de profissionais de saúde. Rev. Eletr. Enferm. [Internet]. 2019 [acesso em: https://doi.org/10.5216/ree.v21.50649. ];21:50649. Disponível em: 


\section{INTRODUÇÃO}

Mesmo não sendo um assunto tão recente, a segurança do paciente ainda é um tema de muito destaque a nível mundial ${ }^{(1)}$. Pois embora o cuidado em saúde traga melhorias a todos os envolvidos, podem ocorrer eventos adversos (EA), os quais são a forma mais inteligível de identificar o erro quantitativamente. Nesse contexto, estima-se que a ocorrência de incidentes relacionados à assistência à saúde, principalmente os EA, afete de 4 a $16 \%$ de pacientes hospitalizados em países desenvolvidos ${ }^{(2)}$.

Nesse contexto, os eventos adversos podem ocorrer em qualquer setor da assistência à saúde, e, especialmente, em Unidades de Terapia Intensiva (UTI), devido à maior complexidade clínica dos pacientes, assim como maior necessidade de procedimentos e dispositivos invasivos, resultando em um maior risco ao paciente, ou seja, em maior vulnerabilidade à ocorrência de $\operatorname{erros}^{(3)}$.

No entanto, para reduzir o número de eventos adversos e melhorar a qualidade da assistência, faz-se fundamental um fortalecimento da cultura de segurança, para que, assim, se possa intervir e promover um compromisso com a segurança do paciente e com um processo de assistência segura ${ }^{(4)}$.

Cultura de segurança é definida como aquela que envolve todos os profissionais, tanto os envolvidos no cuidado como os gestores, encorajando-os a assumirem responsabilidade pela sua própria segurança, pela segurança de seus colegas, pacientes e familiares, priorizando a segurança acima de metas financeiras e operacionais, encorajando e recompensando a identificação, a notificação e a resolução dos problemas relacionados à segurança, além de promover aprendizado a partir do erro e garantir a manutenção dessas estratégias ${ }^{(5)}$. O clima de segurança se refere aos componentes mensuráveis da cultura de segurança, refletindo a concepçáo dos profissionais em relação as questóes de segurança, assim como o comportamento dos gestores ${ }^{(6)}$.

Diante disso, o Safety Attitudes Questionnaire (SAQ) se destaca como um instrumento válido capaz de proporcionar informaçóes em relação aos elementos que necessitam ser implementados na instituição hospitalar para a segurança do paciente, através da mensuração da percepçáo dos profissionais sobre a cultura de segurança, avaliando Clima de trabalho em equipe, Clima de segurança, Satisfação no trabalho, Percepção do estresse, Percepção da gerência e Condiçóes de trabalho ${ }^{(7)}$.

A Associação de Medicina Intensiva Brasileira (AMIB) recomenda, no Guia de Unidades de Terapia Intensiva Seguras (GUTIS), o desenvolvimento de uma cultura de segurança do paciente, principalmente, nessas unidades ${ }^{(8)}$. No entanto, para elaborar açóes voltadas à segurança do paciente, é necessário realizar uma avaliação diagnóstica da cultura de segurança das UTI a fim de conhecer os fatores a serem aprimorados nas unidades (gerenciamento e ambiente de trabalho) e entre os profissionais (estresse, satisfaçáo no trabalho e o clima de trabalho em equipe).

Desse modo, além de justificar a realização desta pesquisa ao cenário assistencial de alto risco das UTI, pouco abordado até então, grande parte dos estudos realizados na temática são do tipo retrospectivo, dificultando a detecção e o registro de eventos adversos e limitando, de forma relevante, a reprodutibilidade das conclusóes encontradas por eles ${ }^{(9)}$.

Assim, a partir da necessidade de estudos sobre cultura de segurança do paciente, especialmente em locais especializados como as Unidades de Terapia Intensiva, surgiu o interesse do grupo de pesquisa Segurança, Tecnologia e Cuidado Clínico da Universidade Estadual do Ceará em realizar esse diagnóstico aplicando o SAQ.

Buscando contribuir para a adoção de uma cultura de segurança do paciente nas instituiçôes de saúde, a partir do diagnóstico situacional sobre o tema em UTIs proporcionado por esse estudo, a presente pesquisa teve como objetivo avaliar a percepção de cultura de segurança do paciente sob a concepçáo dos trabalhadores de Unidades de Terapia Intensiva.

\section{MÉTODOS}

Trata-se de um estudo descritivo-exploratório, transversal, com abordagem quantitativa, desenvolvido em seis Unidades de Terapia Intensiva Adulto e Pediátricas de quatro hospitais públicos da cidade de Fortaleza (CE), referências em neurologia, cardiopneumologia, traumatologia e infectologia.

Os hospitais que participaram do estudo foram escolhidos aleatoriamente. Dos sete hospitais do estado, quatro foram sorteados. $\mathrm{O}$ número de hospitais participantes foi baseado no tempo estimado para a coleta de dados em cada UTI, aproximadamente três meses, por unidade.

A populaçáo foi composta por todos os profissionais de saúde das seis UTIs pesquisadas. Foram incluídos profissionais que possuíam carga horária de trabalho semanal de pelo menos 20 horas e que exerciam suas atividades há pelo menos um mês nas unidades selecionadas para a realização do estudo. Excluíram-se os profissionais afastados de suas atividades por férias ou licença durante o período da coleta de dados ou aqueles que não retornaram o questionário preenchido após três tentativas de contato.

Desta forma, a amostra do estudo foi composta por 283 trabalhadores que obedeceram aos critérios, dentre eles: médicos, enfermeiros, técnicos de enfermagem, fisioterapeutas, nutricionistas, psicólogos, terapeutas ocupacionais, assistentes sociais, farmacêuticos, secretários, funcionários do suporte ambiental e técnicos de encefalograma.

A coleta ocorreu no período de outubro de 2015 a abril de 2016, para que fosse possível abordar uma amostra significativa, utilizando como instrumento o Questionário 
de Atitudes de Segurança - Safety Attitudes Questionnaire (SAQ) —, traduzido e validado para o Brasil, composto por duas partes. A primeira contém 41 questóes, envolvendo a percepçáo sobre segurança do paciente. A segunda parte visa a coletar dados do profissional: cargo exercido, gênero, atuaçáo principal e tempo de atuaçáo.

O SAQ busca avaliar as atitudes de segurança no exercício profissional por meio de seis domínios:

- Clima de Trabalho em Equipe: compreendendo a qualidade do relacionamento e a colaboraçáo entre os membros de uma equipe (itens 1 a 6 );

- Clima de Segurança: avaliação da percepção dos profissionais quanto ao comprometimento organizacional para segurança do paciente (itens 7 a 13);

- Satisfação no Trabalho: visão positiva do local de trabalho (itens 15 a 19);

- Percepçáo do Estresse: reconhecimento do quanto os fatores estressores influenciam na execução do trabalho (itens 20 a 23);

- Percepção da Gerência: aprovação das ações da gerência quanto as questóes de segurança (itens 24 a 29);

- Condiçôes de Trabalho: percepção da qualidade do ambiente de trabalho (itens 30 a 33) ${ }^{(7)}$.

Antes de iniciar a coleta de dados, os pesquisadores se reuniram com as diretorias técnicas e com as chefias dos setores que participaram da pesquisa para compartilhar os objetivos e metodologias do estudo. Os trabalhadores que aceitaram participar da pesquisa assinaram uma via do Termo de Consentimento Livre e Esclarecido (TCLE) e receberam um envelope contendo uma segunda via do TCLE para posse do participante, o instrumento, um lápis e uma borracha. Os instrumentos foram preenchidos pelos participantes no ambiente de trabalho sem a presença do pesquisador, que recolhia os questionários respondidos em outro momento, deixando seu contato disponível para possíveis dúvidas sobre o mesmo. O tempo de resposta do questionário é de, em média, 15 minutos.

O preenchimento das respostas de cada questáo seguiu a escala de cinco pontos de Likert, que varia de discordância total a concordância total, onde foi atribuída uma pontuação a cada opçấo:

- A: discorda totalmente (0 ponto);

- B: discorda parcialmente (25 pontos);

- C: neutro (50 pontos);

- D: concorda parcialmente (75 pontos);

- E: concorda totalmente (100 pontos);

- X: não se aplica.

O escore final varia de a 100, no qual zero corresponde a pior percepçáo de atitudes de segurança pelos profissionais de saúde e 100, a melhor percepção.
Para a pontuaçấo dos escores, as questôes foram ordenadas por domínios, e assim, foi calculada uma média dos escores das questôes de cada domínio. Sendo considerados valores positivos quando o escore final for igual ou maior que 75 pontos.

Após a coleta, os dados foram inseridos em um banco de dados no Excel $^{\circledR}$, e, posteriormente, processados e analisados no Statistical Package for Social Science (SPSS), versão 20.0.

As variáveis qualitativas obtidas pelos questionários foram analisadas através de estatística descritiva por meio da distribuição de frequência absoluta e porcentual. Para a análise das variáveis quantitativas foram utilizadas medidas descritivas de centralidade (média) e de dispersão (desvio padrão, valor mínimo e máximo). Para os testes de comparação de média, adotaram-se a ANOVA e valor de $\mathrm{p}<0,05$.

O estudo foi encaminhado ao Comitê de Ética da Universidade Estadual do Ceará obtendo a aprovação para ser realizado com o número de parecer 985.564. Todos os participantes da pesquisa tiveram o anonimato garantido.

\section{RESULTADOS}

No total, foram distribuídos 470 questionários e 283 $(60,2 \%)$ foram respondidos. Destes, $181(64,4 \%)$ eram da equipe de enfermagem, sendo $118(42,1 \%)$ técnicos/ auxiliares de enfermagem e 63 (22,3\%) enfermeiros. Quanto ao tempo de atuação, constatou-se que 149 (52,7\%) profissionais possuíam mais de cinco anos de trabalho na instituição, prevalecendo os profissionais com vínculo empregatício 119 (42,0\%) (Tabela 1).

Quanto ao escore total do SAQ, observou-se variação entre os quatro hospitais $(63,4$ a 71,5$)$, evidenciando que nenhuma instituição alcançou escore ideal acima de 75 . No que diz respeito à média por domínio, os domínios "Percepção do Estresse" e "Satisfação no Trabalho" alcançaram médias satisfatórias, sendo esse último domínio com diferença estatisticamente significativa entre os hospitais $(p<0,05)$. Em contrapartida, o domínio "Percepçáo da Gerência da Unidade" alcançou médias abaixo de 60 indicando que os profissionais não aprovam as açóes da gerência quanto as questôes de segurança (Tabela 2).

Quanto ao regime de trabalho, observou-se diferença estatisticamente significativa quando comparado a média dos escores dos domínios "Percepção do Estresse" e "Condiçôes de trabalho", entre os profissionais com vínculo empregatício e os profissionais sem o vínculo. No domínio "Condiçôes de Trabalho", observou-se diferença significativa quanto ao tempo de atuação, evidenciando que profissionais com até cinco anos de atuação tendem a avaliar positivamente as condiçôes do ambiente de trabalho.

Quanto à distribuição das respostas dos itens, destacaramse os resultados relacionados ao concordo totalmente e 
parcialmente dos itens 5, 15, 17 e 18, com 83,1\%, 93,4, 85,8 e $85,4 \%$ respectivamente. $\mathrm{O}$ item 5 menciona a facilidade dos profissionais que atuam nas UTIs investigadas a fazerem perguntas quando existe algo que eles não entendem, o item 15 refere-se ao gostar de trabalhar na unidade, indicando que os profissionais estáo satisfeitos com o trabalho, o item 17 aponta as unidades estudadas como um bom lugar para trabalhar e o item 18, o profissional reporta-se ao orgulho

Tabela l. Perfil dos participantes do estudo ( $n=283)$. Fortaleza, CE, Brasil, 2016.

Variáveis $\quad \mathrm{n}(283) \quad \%$

\begin{tabular}{l|c|c}
\multicolumn{1}{l|}{ Gênero } \\
\hline Feminino & 168 & 59,4 \\
\hline Masculino & 98 & 34,6 \\
\hline Não responderam & 17 & 6,0 \\
\hline
\end{tabular}

\begin{tabular}{l|c|c}
\hline \multicolumn{1}{l|}{ Cargo } & 118 & 42,1 \\
\hline Aux/Tec. Enfermagem & 63 & 22,3 \\
\hline Enfermeiro & 27 & 9,5 \\
\hline Médico & 17 & 6,0 \\
\hline Fisioterapeuta & 11 & 3,9 \\
\hline Nutricionista & 7 & 2,5 \\
\hline Médico Residente & 6 & 2,1 \\
\hline Chefe de Enfermagem & 5 & 1,8 \\
\hline Terapeuta Ocupacional & 4 & 1,4 \\
\hline Farmacêutico & 3 & 1,0 \\
\hline Psicólogo & 2 & 0,7 \\
\hline Assistente Social & 11 & 3,9 \\
\hline Outros & 9 & 3,2 \\
\hline Não responderam & &
\end{tabular}

\begin{tabular}{l|c|c}
\hline \multicolumn{1}{l|}{ Tempo de atuação } & 17 & 6,0 \\
\hline Menor que 6 meses & 16 & 5,7 \\
\hline 6 a ll meses & 46 & 16,2 \\
\hline l a 2 anos & 32 & 11,3 \\
\hline 3 a 4 anos & 59 & 20,9 \\
\hline 5 a 10 anos & 57 & 20,1 \\
\hline 1l a 20 anos & 33 & 11,7 \\
\hline 2l anos ou mais & 23 & 8,1 \\
\hline Não responderam & \multicolumn{2}{|l}{} \\
\hline Regime de trabalho & 119 & 42,0 \\
\hline Com vínculo & 107 & 37,8 \\
\hline Sem vínculo & 29 & 10,2 \\
\hline Outros & 28 & 10,0 \\
\hline Não responderam
\end{tabular}

de trabalhar na unidade. Ressalta-se, ainda, as repostas positivas dos itens 20 e 33 dos domínios "Percepção do Estresse" e "Condiçóes de Trabalho", respectivamente. Essas questôes evidenciam que $79,7 \%$ dos profissionais concordam que quando a carga de trabalho é excessiva o desempenho é prejudicado e $83,4 \%$ concordam que vivenciam uma boa colaboração com os enfermeiros da unidade (Tabela 3).

No que se refere à gerência da unidade e do hospital, o item 24 "A administração apoia meus esforços diários" e o item 25 "A administração não compromete conscientemente a segurança do paciente" indicaram média de 40,3 e 41,9\%, respectivamente, de respostas negativas.

\section{DISCUSSÃO}

Os resultados variaram de acordo com os domínios, os hospitais, regime de trabalho e tempo de atuação.

A maioria dos profissionais das UTI estudadas é da equipe de enfermagem, com tempo de atuação maior que cinco anos e com vínculo com a instituição. Esses resultados são semelhantes aos dados identificados no estudo realizado pelo departamento de Enfermagem da Associação de Medicina Intensiva no qual revelou que os profissionais brasileiros que atuam em UTI são predominantemente do sexo feminino, com idade média em torno dos 35 anos e vinculados a um regime trabalhista ${ }^{(10)}$.

Quanto à percepção da cultura de segurança, nenhuma instituição alcançou o resultado acima de 75. Esse resultado assemelha-se com o de um estudo realizado em UTI internacional ${ }^{(11)}$, bem como os de outros estudos brasileiros ${ }^{(12,13)}$, que também revelaram índices baixos e uma cultura de segurança frágil em UTI na maioria das dimensóes avaliadas.

O impacto da cultura de segurança no cuidado de saúde e na segurança do trabalhador tem sido objeto de estudo desde a década de 1990 . Um estudo realizado no Brasil ${ }^{(14)}$ revela a importância das pesquisas realizadas em unidades especificas, como em UTI, para avaliar as condiçóes de um ambiente de trabalho que podem levar a eventos adversos e danos ao paciente. Acredita-se que este tipo de avaliação pode aumentar a conscientização sobre questóes de segurança do paciente, avaliar o status atual da cultura de segurança e acompanhar a eficácia das intervençóes ao longo do tempo.

Quanto aos domínios, a "Satisfação no Trabalho" obteve maior média, com variação entre 71 a 84 e diferença estatisticamente significativa entre hospitais. A UTI do hospital A que obteve maior média nesse domínio, é uma unidade composta em sua maioria por profissionais que passaram por processo de concurso público, ou seja, possuem vínculo permanente com a instituição. $\mathrm{O}$ oposto é observado na UTI do hospital $\mathrm{C}$ que possui o maior número de profissionais sem o vínculo com a instituição. Não podemos afirmar que o vínculo com a instituição tem associação direta 
com a satisfação no trabalho, no entanto esses resultados podem indicar fatores que eventualmente tenha relação com esse construto.

Salienta-se que as diversas modalidades de vinculação ao trabalho apontam para uma precarização de um contingente significativo de trabalhadores da saúde, tal precarização se constitui como um ponto crítico para a consolidaçáo do SUS e para a garantia do direito à saúde ${ }^{(15)}$. A ausência do vínculo com a instituição compromete a relação com os trabalhadores e prejudica a continuidade dos serviços prestados, pois tem como consequência o alto índice de rotatividade dos profissionais ${ }^{(16)}$. Estudos constataram que essa rotatividade pode estar associada com a satisfação no trabalho ${ }^{(10)}$ e com resultados relacionados ao paciente, como o tempo de permanência e incidentes que constituem uma ameaça à segurança e ao bem-estar dos paciente ${ }^{(17)}$.

Ainda quanto ao regime de trabalho, observou-se que o domínio "Percepção do Estresse" apresenta média estatisticamente significativa entre os profissionais com vínculo e sem vínculo com a instituição. A interpretação desses resultados reforça os resultados do domínio "Satisfação no Trabalho", uma vez que profissionais com vínculo e satisfeitos com a instituiçáo sentem-se mais seguros e conseguem reconhecer o quanto os fatores estressores interferem na execução do trabalho. Com esses achados, percebe-se que os participantes têm consciência de que fatores como carga excessiva de trabalho, situaçóes estressantes e cansaço podem comprometer a segurança do paciente e gerar situaçóes propícias à ocorrência de eventos adversos.

O excesso da carga de trabalho é visto como responsável pelo desgaste emocional, ocorrência de acidentes e problemas de saúde dos profissionais, devendo existir dessa maneira um planejamento adequado da distribuição de carga de trabalho, educação continuada, elaboração de estratégias para melhoria das condiçóes de trabalho, com o intuito de prevenir o desgaste físico e psíquico da equipe ${ }^{(18)}$.

Estudos demonstram a influência da satisfação no trabalho na qualidade dos cuidados aos pacientes. Sendo ressaltado que se

Tabela 2. Escores do Safety Attitudes Questionnaire (SAQ) por domínio de acordo com hospital, regime de tra-

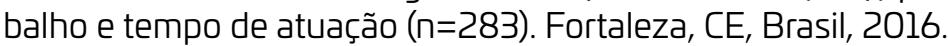

\begin{tabular}{|c|c|c|c|c|c|c|c|c|}
\hline \multirow{2}{*}{ Variáveis } & \multicolumn{7}{|c|}{ Domínios do SAQ } & \multirow{2}{*}{ SAQ Total } \\
\hline & $\mathrm{Dl} \cdot$ & D2· & D3· & D4. & D5A• & D5B· & D6. & \\
\hline Hospital & Média & Média & Média & Média & Média & Média & Média & Média \\
\hline A & $82,0^{*}$ & 65,5 & $84,0^{*}$ & 78,6 & 63,7 & 58,2 & 65,2 & 71,5 \\
\hline B & $69,6^{*}$ & 59,9 & 76,0 & 75,6 & 53,4 & 52,1 & 68,4 & 64,6 \\
\hline C & 69,8 & 59,8 & $71,7^{*}$ & 71,3 & 53,6 & 48,4 & 67,5 & 63,8 \\
\hline $\mathrm{D}$ & 73,4 & 66,7 & 78,9 & 74,8 & 54,3 & 57,7 & 52,4 & 63,4 \\
\hline
\end{tabular}

Regime de trabalho

\begin{tabular}{l|c|c|c|c|c|c|c|c}
\hline Com vínculo & 73,1 & 60,0 & 76,4 & $83,1^{*}$ & 55,1 & 47,8 & $59,2^{\star}$ & 65,2 \\
\hline Sem vínculo & 70,0 & 63,2 & 75,9 & 68,1 & 55,5 & 54,5 & 68,3 & 65,3 \\
\hline Outros & 76,9 & 67,1 & 79,9 & 74,2 & 61,4 & 51,7 & 70,0 & 69,4 \\
\hline
\end{tabular}

Tempo de atuação

\begin{tabular}{l|l|l|l|l|l|l|l|l}
\hline Menos de 6 meses & 80,8 & 73,9 & 79,1 & 68,7 & 65,8 & 63,2 & $75,7^{\star}$ & 73,3 \\
\hline 6 a ll meses & 70,3 & 61,8 & 72,9 & 79,9 & 52,8 & 52,1 & 71,6 & 66,4 \\
\hline la 2 anos & 74,2 & 63,8 & 77,2 & 74,1 & 58,5 & 52,6 & 70,7 & 67,8 \\
\hline 3 a 4 anos & 72,2 & 63,9 & 78,8 & 75,0 & 61,0 & 60,6 & 72,4 & 69,0 \\
\hline 5 a 10 anos & 72,6 & 62,4 & 74,4 & 72,0 & 52,5 & 47,8 & 63,0 & 63,6 \\
\hline 11 a 20 anos & 71,7 & 59,1 & 76,8 & 81,1 & 56,0 & 45,7 & 59,0 & 64,7 \\
\hline Maior que 21 anos & 70,5 & 62,1 & 77,9 & 69,2 & 51,8 & 51,2 & 60,1 & 63,7 \\
\hline
\end{tabular}

•Dl: clima de trabalho em equipe; D2: clima de segurança; D3: satisfação no trabalho; D4: percepção do estresse; D5A: percepção da gerência do hospital; D5B: percepção da gerência da unidade; D6: condição de trabalho; * ${ }^{*}<0,05$. 
Tabela 3. Distribuição das respostas dos participantes por item ( $n=283)$. Fortaleza, CE, Brasil, 2016.

\begin{tabular}{|c|c|c|c|}
\hline \multirow[t]{2}{*}{ SAO } & $\begin{array}{l}\text { Discordo totalmente } \\
\text { e parcialmente }\end{array}$ & Neutro & $\begin{array}{c}\text { Concordo } \\
\text { totalmente e } \\
\text { parcialmente }\end{array}$ \\
\hline & $n(\%)$ & $n(\%)$ & n (\%) \\
\hline $\begin{array}{l}\text { l. As sugestões do(a) enfermeiro(a) são bem recebidas } \\
\text { nesta área. }\end{array}$ & $30(10,9)$ & $34(12,3)$ & $212(76,8)$ \\
\hline $\begin{array}{l}\text { 2. (R)* Nesta área, é difícil falar abertamente se eu percebo } \\
\text { um problema com o cuidado ao paciente. }\end{array}$ & $121(44,3)$ & $23(8,4)$ & $129(47,3)$ \\
\hline $\begin{array}{l}\text { 3. Nesta área, as discordâncias são resolvidas de modo } \\
\text { apropriado (ex.: não quem está certo, mas o que é melhor } \\
\text { para o paciente). }\end{array}$ & $42(15,2)$ & $29(10,5)$ & $205(74,3)$ \\
\hline $\begin{array}{l}\text { 4. Eu tenho o apoio que necessito de outros membros da } \\
\text { equipe para cuidar dos pacientes. }\end{array}$ & $25(9,1)$ & $29(10,6)$ & $219(80,2)$ \\
\hline $\begin{array}{l}\text { 5. É fácil para os profissionais que atuam nesta área fazerem } \\
\text { perguntas quando existe algo que eles não entendem. }\end{array}$ & $30(11,0)$ & $16(5,9)$ & $227(83,1)$ \\
\hline $\begin{array}{l}\text { 6. Os(as) médicos(as) e enfermeiros(as) daqui trabalham } \\
\text { juntos como uma equipe bem coordenada. }\end{array}$ & $43(15,7)$ & $20(7,3)$ & $210(77,0)$ \\
\hline $\begin{array}{l}\text { 7. Eu me sentiria seguro(a) se fosse tratado(a) aqui como } \\
\text { paciente. }\end{array}$ & $37(13,6)$ & $28(10,3)$ & $207(76,1)$ \\
\hline 8. Erros são tratados de modo apropriado nesta área. & $62(22,6)$ & $45(16,4)$ & $168(61,1)$ \\
\hline $\begin{array}{l}\text { 9. Eu conheço os meios adequados para encaminhar as } \\
\text { questões relacionadas à segurança do paciente nesta área. }\end{array}$ & $46(16,7)$ & $36(13,0)$ & $194(70,3)$ \\
\hline 10. Eu recebo retorno apropriado sobre meu desempenho. & $106(39,3)$ & $45(16,7)$ & $119(44,0)$ \\
\hline 11. (R)* Nesta área, é difícil discutir sobre erros. & $126(45,6)$ & $50(18,1)$ & $100(36,2)$ \\
\hline $\begin{array}{l}\text { 12. Sou encorajado(a) por meus colegas a informar qualquer } \\
\text { preocupação que eu possa ter quanto à segurança do paciente. }\end{array}$ & $45(16,5)$ & $42(15,4)$ & $185(68,0)$ \\
\hline $\begin{array}{l}\text { 13. A cultura nesta área torna fácil aprender com os erros } \\
\text { dos outros. }\end{array}$ & $69(25,4)$ & $48(17,7)$ & $154(56,9)$ \\
\hline $\begin{array}{l}\text { 14. Minhas sugestões sobre segurança seriam postas em } \\
\text { ação se eu as expressasse à administração. }\end{array}$ & $75(28,3)$ & $63(23,8)$ & $127(47,9)$ \\
\hline 15. Eu gosto do meu trabalho. & $5(1,8)$ & $13(4,8)$ & $255(93,4)$ \\
\hline 16. Trabalhar aqui é como fazer parte de uma grande família. & $27(9,9)$ & $33(12,1)$ & $213(78,0)$ \\
\hline 17. Este é um bom lugar para trabalhar. & $14(5,1)$ & $25(9,1)$ & $237(85,8)$ \\
\hline 18. Eu me orgulho de trabalhar nesta área. & $17(6,2)$ & $23(8,4)$ & $234(85,4)$ \\
\hline 19. 0 moral nesta área é alto. & $59(22,6)$ & $51(19,5)$ & $152(58,0)$ \\
\hline $\begin{array}{l}\text { 20. Quando minha carga de trabalho é excessiva, meu } \\
\text { desempenho é prejudicado. }\end{array}$ & $35(12,9)$ & $20(7,4)$ & $216(79,7)$ \\
\hline $\begin{array}{l}\text { 21. Eu sou menos eficiente no trabalho quando estou } \\
\text { cansado(a). }\end{array}$ & $38(14,0)$ & $20(7,4)$ & $213(78,6)$ \\
\hline $\begin{array}{l}\text { 22. Eu tenho maior probabilidade de cometer erros em } \\
\text { situações tensas ou hostis. }\end{array}$ & $47(17,4)$ & $26(9,6)$ & $197(73,0)$ \\
\hline $\begin{array}{l}\text { 23. O cansaço prejudica meu desempenho durante situações de } \\
\text { emergência (ex: reanimação cardiorrespiratória, convulsões). }\end{array}$ & $61(24,4)$ & $28(11,2)$ & $162(64,5)$ \\
\hline 24. A administração apoia meus esforços diários: (Hospital). & $58(32,0)$ & $27(14,9)$ & $96(53,0)$ \\
\hline
\end{tabular}


Tabela 3. Continuação.

\begin{tabular}{|c|c|c|c|}
\hline \multirow[t]{2}{*}{ SAQ } & $\begin{array}{l}\text { Discordo totalmente } \\
\text { e parcialmente }\end{array}$ & Neutro & $\begin{array}{c}\text { Concordo } \\
\text { totalmente e } \\
\text { parcialmente }\end{array}$ \\
\hline & n (\%) & $n(\%)$ & $n(\%)$ \\
\hline 24. A administração apoia meus esforços diários: (Unidade). & $90(40,3)$ & $61(27,4)$ & $72(32,0)$ \\
\hline $\begin{array}{l}\text { 25. A administração não compromete conscientemente a } \\
\text { segurança do paciente: (Hospital) }\end{array}$ & $75(41,9)$ & $45(25,1)$ & $59(32,9)$ \\
\hline $\begin{array}{l}\text { 25. A administração não compromete conscientemente a } \\
\text { segurança do paciente: (Unidade) }\end{array}$ & $62(27,7)$ & $76(33,9)$ & $86(38,4)$ \\
\hline 26. A administração está fazendo um bom trabalho: (Hospital) & $38(20,5)$ & $37(20)$ & $110(59,5)$ \\
\hline 26. A administração está fazendo um bom trabalho: (Unidade) & $57(25,1)$ & $72(31,7)$ & $98(43,2)$ \\
\hline $\begin{array}{l}\text { 27. Profissionais problemáticos da equipe são tratados de } \\
\text { maneira construtiva por nossa: (Hospital) }\end{array}$ & $58(31,2)$ & $58(31,2)$ & $70(37,6)$ \\
\hline $\begin{array}{l}\text { 27. Profissionais problemáticos da equipe são tratados de } \\
\text { maneira construtiva por nossa: (Unidade) }\end{array}$ & $71(32,3)$ & $75(34,1)$ & $74(33,7)$ \\
\hline $\begin{array}{l}\text { 28. Recebo informações adequadas e oportunas sobre } \\
\text { eventos que podem afetar meu trabalho do(a): (Hospital) }\end{array}$ & $57(30,8)$ & $34(18,4)$ & $94(50,8)$ \\
\hline $\begin{array}{l}\text { 28. Recebo informações adequadas e oportunas sobre } \\
\text { eventos que podem afetar meu trabalho do(a): (Unidade) }\end{array}$ & $71(32,3)$ & $60(27,3)$ & $89(40,4)$ \\
\hline $\begin{array}{l}\text { 29. Nesta área, o número e a qualificação dos profissionais } \\
\text { são suficientes para lidar com o número de pacientes. }\end{array}$ & $118(4,4)$ & $32(12)$ & $116(43,6)$ \\
\hline $\begin{array}{l}\text { 30. Este hospital faz um bom trabalho no treinamento de } \\
\text { novos membros da equipe. }\end{array}$ & $69(25,9)$ & $39(14,7)$ & $158(59,4)$ \\
\hline $\begin{array}{l}\text { 31. Toda informação necessária para decisões diagnósticas } \\
\text { e terapêuticas está disponível rotineiramente para mim. }\end{array}$ & $69(25,7)$ & $44(16,4)$ & $156(58,0)$ \\
\hline $\begin{array}{l}\text { 32. Estagiários da minha profissão são adequadamente } \\
\text { supervisionados. }\end{array}$ & $52(19,7)$ & $26(9,8)$ & $186(70,5)$ \\
\hline $\begin{array}{l}\text { 33. Eu vivencio boa colaboração com os (as) enfermeiros(as) } \\
\text { nesta área. }\end{array}$ & $28(10,3)$ & $17(6,3)$ & $226(83,4)$ \\
\hline $\begin{array}{l}\text { 34. Eu vivencio boa colaboração com a equipe de médicos } \\
\text { nesta área. }\end{array}$ & $34(12,5)$ & $26(9,5)$ & $213(78,0)$ \\
\hline $\begin{array}{l}\text { 35. Eu vivencio boa colaboração com os farmacêuticos } \\
\text { nesta área. }\end{array}$ & $50(19,0)$ & $44(16,8)$ & $168(64,1)$ \\
\hline $\begin{array}{l}\text { 36. }(\mathrm{R})^{\star} \text { Falhas na comunicação que levam a atrasos no } \\
\text { atendimento são comuns. }\end{array}$ & $152(57,4)$ & $46(17,4)$ & $67(25,3)$ \\
\hline
\end{tabular}

SAQ: Safety Attitudes Questionnaire; *itens reversos.

há insatisfação com o trabalho, por pelo menos parte da equipe, toda a equipe de profissionais pode ser afetada, diminuindo a qualidade da assistência ao paciente e provocando, assim, prolongamento do tempo de permanência hospitalar, aumento do número de eventos adversos e mortalidade ${ }^{(19)}$, além de ter associação com desempenho, motivação, absentismo/atraso, saúde mental/física e satisfação geral da vida ${ }^{(20)}$.

Destacaram-se também os domínios "Percepção da Gerência", "Condições de Trabalho" e "Clima de segurança”, os quais obtiveram escores abaixo do esperado, variando entre 48,4 a 58,$2 ; 52,4$ a 68,4 e 59,9 a 66,7 , respectivamente. A percepção da gerência expressa a aprovação por parte dos profissionais quanto às açóes da gerência relacionadas às questóes de segurança do paciente. Resultados semelhantes foram identificados em estudos com o SAQ na Itália ${ }^{(21)}$ e no Brasil $^{(14)}$.

As percepçóes negativas da gerência refletem o descontentamento dos profissionais com os seus gestores quanto 
as questóes de segurança do paciente, e estas ficam evidentes quando os profissionais referem que não se sentem apoiados em seus esforços diários. Dessa forma, os profissionais podem sentir-se menos valorizados em seu trabalho, com impacto no atendimento ao paciente. No entanto, vale salientar que nas unidades de terapia intensiva o perfil clínico dos pacientes e alta mortalidade pode levar ao esgotamento pessoal e ao estresse, o que pode refletir na percepção da gestáo hospitalar.

Um estudo brasileiro também analisou os incidentes em UTIs e revelou influência das características do trabalho dos profissionais de UTI e dos níveis de estresse, burnout, carga de trabalho de enfermagem, satisfação profissional e percepção do ambiente de trabalho na segurança dos pacientes $^{(22)}$.

Quanto ao domínio "Condições de Trabalho", obteve-se escore $>75$ com diferença estatisticamente significativa para os profissionais com tempo de atuação menor que seis meses. Esse resultado pode ser explicado pelo fato desses profissionais estarem se adaptando ao novo local de trabalho, diferente do profissional que está há mais de 10 anos na instituição e que espera-se possuir uma visão mais crítica do ambiente de trabalho. No entanto, estudo realizado no Brasil ${ }^{(12)}$ revelou que o tempo de experiência no trabalho não teve diferença significativa quanto a uma melhor percepção de cultura de segurança.

Nesse estudo, o "Clima de Segurança” é um dos domínios avaliados pelo instrumento e não foi correlacionado com variável alguma do estudo. A percepção de um forte e proativo compromisso organizacional com a segurança obteve média abaixo do esperado, no entanto quando avaliado os itens desse domínio observa-se que a maioria dos profissionais se sentiriam seguros se fossem pacientes daquelas unidades, além disso, mais de $70 \%$ referiu conhecer os meios adequados para encaminhar as questóes relacionadas à segurança do paciente.

Tal fato condiz com a proposta de uma cultura de segurança do paciente robusta, a qual busca uma compreensão de toda a equipe multiprofissional, que assiste direta e indiretamente ao paciente, de que as necessidades e os erros devem ser expostos e discutidos claramente entre os profissionais, usando uma abordagem não-punitiva, incentivando os profissionais a notificar os eventos ocorridos, para que a instituição possa intervir nos processos de trabalho e na formação permanente, empoderando-os para garantir uma cultura da segurança e uma assistência mais segura ${ }^{(23)}$.

Ressalta-se que o presente estudo, avaliou a cultura de segurança em um tempo determinado e náo analisou a correlação da cultura de segurança com indicadores de segurança do paciente, sem identificar o impacto da cultura de segurança em tais indicadores. Novas investigaçóes com outros delineamentos de pesquisa ampliarão o conhecimento sobre o assunto e minimizarão as limitaçóes citadas.

\section{CONCLUSÃO}

A partir dos resultados dessa pesquisa, observa-se que a percepção de segurança do paciente dos trabalhadores das unidades estudadas apresentou escores abaixo do esperado $(<75)$, refletindo uma cultura de segurança que precisa ser melhorada em diversos aspectos, especialmente em atitudes gerenciais quanto à segurança do paciente. Devem também ser fortalecidos aspectos como percepçáo do estresse e condiçóes de trabalho.

Além disso, observou-se que os profissionais com vínculo empregatício perceberam melhor os fatores que levam ao estresse e são mais críticos quanto às condiçóes de trabalho, evidenciando a importância da efetivação do vínculo empregatício dos profissionais da saúde para uma assistência segura.

De acordo com o que foi obtido no estudo, revelou-se a necessidade de se trabalhar os aspectos gerenciais dentro do ambiente de trabalho, especialmente em UTI, por serem locais de prestação de cuidados complexos a pacientes críticos. Sugere-se que a cultura de segurança seja avaliada continuamente, de modo que os dados possam auxiliar os gerentes a traçarem estratégias para a implantação de uma cultura justa, na qual as pessoas não são punidas, mas as violações não são toleradas, a Cultura de relato e notificação e a Cultura de aprendizagem contínua, na qual os erros devem ser conhecidos, investigados, as soluçóes encontradas e implementadas.

Com o diagnóstico realizado, tal estudo serve como um ponto de partida para o início de mudanças de políticas para abordagem dos problemas identificados. Incentivando, assim, a implementação de intervençóes futuras direcionadas a reduzir o impacto desses fatores sobre a qualidade da assistência e segurança do paciente.

\section{REFERÊNCIAS}

1. Minuzzi AP, Salum NC, Locks $\mathrm{MOH}$, Amante LN, Matos E. Contribuiçóes da equipe de saúde visando à promoção da segurança do paciente no cuidado intensivo. Esc. Anna Nery [Internet]. 2016 [acessado em 28 nov. 2018];20(1):121-9. Disponível em: http:// dx.doi.org/10.5935/1414-8145.20160017.

2. Brasil. Ministério da Saúde. Agência Nacional de Vigilância Sanitária. Gestão de Riscos e Investigação de Eventos Adversos Relacionados à Assistência à Saúde [Internet]. Brasília: Ministério da Saúde; 2017 [acessado em 20 nov. 2018]. Disponível em: http:// portal.anvisa.gov.br/documents/33852/3507912/ Caderno+7++Gest $\% \mathrm{C} 3 \% \mathrm{~A} 3 \mathrm{o}+\mathrm{de}+\mathrm{Riscos}+\mathrm{e}+$ Invest iga $\% \mathrm{C} 3 \% \mathrm{~A} 7 \% \mathrm{C} 3 \% \mathrm{~A} 3 \mathrm{o}+\mathrm{de}+$ Eventos + Adversos $+\mathrm{R}$ elacionados+ $\% \mathrm{C} 3 \% \mathrm{~A} 0+$ Assist $\% \mathrm{C} 3 \% \mathrm{AAncia}+\% \mathrm{C}$ 3\%A0+Sa\%C3\%BAde/6fa4fa91-c652-4b8b-b56efe466616bd57. 
3. Ortega DB, D’innocenzo M, Silva LMG, Bohomol E. Análise de eventos adversos em pacientes internados em unidade de terapia intensiva. Acta Paul Enferm [Internet]. 2017 [acessado em 19 nov. 2018];30(2):16873. Disponível em: http://dx.doi.org/10.1590/19820194201700026.

4. Roque KE, Tonini T, Melo ECP. Eventos adversos na unidade de terapia intensiva: impacto na mortalidade e no tempo de internação em um estudo prospectivo. Cad Saúde Pública [Internet]. 2016 [acessado em 22 nov. 2018];32(10):e00081815. Disponível em: http:// dx.doi.org/10.1590/0102-311X00081815.

5. Brasil. Ministério da Saúde. Fundação Oswaldo Cruz. Agência Nacional de Vigilância Sanitária. Documento de referência para o Programa Nacional de Segurança do Paciente [Internet]. Brasília: Ministério da Saúde; 2014 [acessado em 13 out. 2018]. Disponível em: http:// bvsms.saude.gov.br/bvs/publicacoes/documento referencia_programa_nacional_seguranca.pdf.

6. Kolankiewicz AC, Loro MM, Schmidt CR, Santos FP, Bandeira VAC, Magnago TSBS. Clima de segurança do paciente entre trabalhadores de enfermagem: fatores contribuintes. Acta Paul Enferm [Internet]. 2017 [acessado em 20 nov. 2018];30(5):531-7. Disponível em: http://dx.doi.org/10.1590/1982-0194201700076.

7. Carvalho REFL, Arruda LP, Nascimento NKP, Sampaio RL, Cavalcante MLSN, Costa ACP. Avaliação da cultura de segurança em hospitais públicos no Brasil. Rev Latino-Am Enfermagem [Internet]. 2017 [acessado em 20 nov. 2018];25:e2849. Disponível em: http:// dx.doi.org/10.1590/1518-8345.1600.2849.

8. Tomazoni A, Rocha PK, Kusahara DM, Souza AIJ, Macedo TR. Avaliaçáo da cultura de segurança do paciente em terapia intensiva neonatal. Texto Contexto Enferm. 2015 [acessado em 22 nov. 2018];24(1):1619. Disponível em: http://dx.doi.org/10.1590/010407072015000490014.

9. Novaretti MCZ, Santos EV, Quitério LM, DaudGallotti RM. Sobrecarga de trabalho da Enfermagem e incidentes e eventos adversos em pacientes internados em UTI. Rev Bras Enferm [Internet]. 2014 [acessado em 15 out. 2018];67(5):692-9. Disponível em: http:// dx.doi.org/10.1590/0034-7167.2014670504.

10. Viana RAPP, Vargas MAO, Carmagnani MIS, Tanaka LH, Luz KR, Schmitt PH. Profile of an intensive care nurse in different regions of Brazil. Texto Contexto Enferm [Internet]. 2014 [acessado em 11 out. 2018];23(1):151-9. Disponível em: http://dx.doi. org/10.1590/S0104-07072014000100018.

11. Elsous A, Sari AA, Rashidian A, Aljeesh Y, Radwan M, AbuZaydeh H. A cross-sectional study to assess the patient safety culture in the Palestinian hospitals: a baseline assessment for quality improvement. JRSM Open [Internet]. 2016 [acessado em 15 out. 2018];7(12):167-75. Disponível em: https://doi. org/10.1177/2054270416675235.

12. Tomazoni A, Rocha PT, Souza S, Anders JC, Malfussi HFC. Cultura de segurança do paciente em unidades de terapia intensiva neonatal: perspectivas da equipe de enfermagem e médica. Rev Lat Am Enferm [Internet]. 2014 [acessado em 16 out. 2018];22(5):755-63. Disponível em: https://doi.org/10.1590/01041169.3624.2477.

13. Mello JF, Barbosa SFF. Cultura de segurança do paciente em unidade de terapia intensiva: perspectiva da equipe de enfermagem. Rev Eletr Enf [Internet]. 2017 [acessado em 13 out. 2018];19(7):2-12. Disponível em: https://doi.org/10.5216/ree.v19.38760.

14. Santiago THR, Turrini RNT. Cultura e clima organizacional para segurança do paciente em Unidades de Terapia Intensiva. Rev Esc Enferm USP [Internet]. 2015 [acessado em 17 out. 2018];49(Núm. Esp.):12330. Disponível em: http://dx.doi.org/10.1590/S0080623420150000700018.

15. Eberhardt LD, Carvalho M, Murofuse NT. Vínculos de trabalho no setor saúde: o cenário da precarizaçáo na macrorregiẫo Oeste do Paraná. Saúde em Debate [Internet]. 2015 [acessado em 17 out. 2018];39(104):1829. Disponível em: http://dx.doi.org/10.1590/0103110420151040432.

16. Pialarissi R. Precarização do Trabalho. Rev Adm Saúde [Internet]. 2017 [acessado em 21 nov. 2018];17(66). Disponível em: http://dx.doi.org/10.23973/ras.66.11.

17. Leitão IMTA, Sousa FSP, Santiago JCS, Bezerra IC, Morais JB. Absenteeism, turnover, and indicators of quality control in nursing care: a transversal study. Online Braz J Nurs [Internet]. 2017 [acessado em 17 out. 2018];16(2):119-29. Disponível em: https://doi. org/10.17665/1676-4285.20175623.

18. Chiang HY, Hsiao YC, Lee HF. Predictors of Hospital nurse's Safety Pratices: Work Environment, Workload, Job satisfaction, and Error Reporting. J Nurs Care Qual [Internet]. 2017 [acessado em 12 out. 2018];32(4):35968. Disponível em: https://doi.org/10.1097/ NCQ.0000000000000240.

19. Bordignon M, Monteiro MI, Mai S, Martins MFSV, Rech CRA, Trindade LL. Satisfação e insatisfação no trabalho de profissionais de enfermagem da oncologia do Brasil e Portugal. Texto Contexto Enferm [Internet]. 2015 [acessado em 22 nov. 2018];24(4):925-33. Disponível em: http://dx.doi.org/10.1590/01040707201500004650014.

20. Körner M, Wirtz MA, Bengel J, Göritz AS. Relationship of organizational culture, teamwork 
and job satisfaction in interprofessional teams. BMC Health Serv Res [Internet]. 2015 [acessado em 15 out. 2018];15:243. Disponível em: https://doi. org/10.1186/s12913-015-0888-y.

21. Zenere A, Zanolin ME, Negri R, Moretti F, Grassi M, Tardivo S. Assessing safety culture in NICU: psychometric properties of the Italian version of Safety Attitude Questionnaire and result implications. J Eval Clin Pract [Internet]. 2016 [acessado em 15 out. 2018];22(2):275-82. Disponível em: https://doi. org/10.1111/jep. 12472 .

22. Padillha KG, Barbosa RL, Andolhe R, Oliveira EM, Ducci AJ, Bregalda RS, et al. Carga de trabalho de enfermagem, estresse/burnout, satisfação e incidentes em unidade de terapia intensiva de trauma. Texto Contexto Enferm [Internet]. 2017 [acessado em 20 dez. 2018];26(3):e1720016. Disponível em: http://dx.doi.org/10.1590/010407072017001720016.

23. Lemos GC, Azevedo C, Bernardes MFGV, Ribeiro HCTC, Menezes AC, da Mata LRF. A cultura de segurança do paciente no âmbito da enfermagem: reflexão teórica. Rev Enferm Centro-Oeste Mineiro [Internet]. 2018 [acessado em 20 nov. 2018];8:e2600. Disponível em: http://dx.doi.org/10.19175/recom. v8i0.2600. 